\title{
阿哈湖 $\mathrm{Fe} 、 \mathrm{Mn}$ 沉积后再迁移 的生物地球化学机理
}

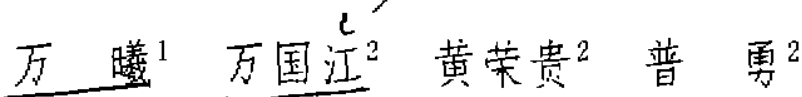

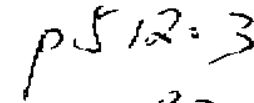

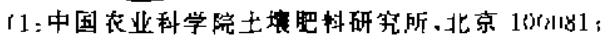

2: 中国科学院地球化学研究所环境地球化学国家重点实验宣，费附 5511100 )

提要进河除湖是一底层滞水带季节性缺氧的中型人工湖. 由于长期积累. 沉积物顶部 微粒悬浮层出现了 $\mathrm{Fe} 、 \mathrm{Mn}$ 富集. 湖底缺氧时，经生物氧化作用形成的 $\mathrm{Fe}^{\mathrm{i}} 、 \mathrm{Mn}^{2+}$ 自沉积物向上影 水体扩散、水体 $\mathrm{Fe}^{2+}$ 㳖度增高比 $\mathrm{Mn}^{2+}$ 滞后出现且超过前结束.沉积物中硫酸盐迈原作用一般在沉 积物一水界面以下 $6 \mathrm{~cm}$ 深度内进行、但在缺氧季节上移至沉积物顶部，并抑制了 $\mathrm{Fe}^{2+}$ 的释放. 还源 态 $\mathrm{Mn}^{\mathrm{i}+}$ 的氧化作用需分子氧参与、缺氧导致 $\mathrm{Mn}$ 的扩散这移. 基于天然水体中存在聚果 $\mathrm{Mn}$ 的生

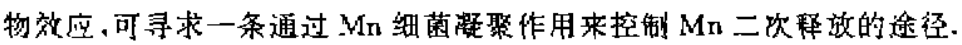

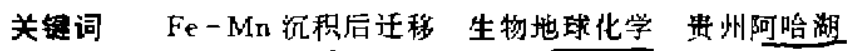

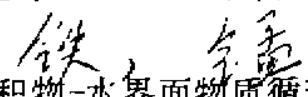

湖泊沉积物一水界面物质循环过程中微生物活动具有重要意义，表层沉积物中的大部分有 机质经生物氧化作用被分解、形成还原性化合物 $\mathrm{CH}_{4} 、 \mathrm{NH}_{4}{ }^{+} 、 \mathrm{H}_{2} \mathrm{~S} 、 \mathrm{Fe}^{2+} 、 \mathrm{Mn}{ }^{2+}+$ 等. 湖水季节性 $\mathrm{Fe}, \mathrm{Mn}$ 沉积后迁移便是沉积物一水界面氧化还原条件变化的结果 ${ }^{[-4]}$.

贵州阿哈湖位于贵阳市西南郊 $8 \mathrm{~km}$, 是一底层滞水带季节性缺氧的中型人工湖、兼具蓄 水、供水和防洪功能.该湖于 1958 年建坝修库、1960 年竣工蓄水.1982 年扩大蓄水面积。并开 发为城市饮用水源. 湖区汇水面积 $190 \mathrm{~km}^{2}$, 湖水面积 $3.4 \mathrm{~km}^{2}$, 平均水深 $13 \mathrm{~m}$.最大水深 $24 \mathrm{~m}$. 湖水容量 $0.445 \times 10^{8} \mathrm{~m}^{3}$ 、水输送通量 $1.04 \times 10^{\mathrm{B}} \mathrm{m}^{3} / \mathrm{a}$ 、湖水寄宿时间 $0.44 \mathrm{a}$. 阿哈湖集水区域主 要分布二迭系灰岩及煤系地层,其上发育硅铝质和硅铁质黄壤; 部分地区有三迭系碳酸盐类岩 石及泥页岩出露、并发育着黑色、黄褐色石灰土、湖周为疏林植被、灌木丛、灌草丛较多、流域年 均降水量 $1109 \mathrm{~mm}$,年均气温 $15.3 \mathrm{C}$.

阿哈湖集水区域分布有中小煤矿 200 余个、入湖河流每年纳入煤矿排出含 $\mathrm{Fe} 、 \mathrm{Mn}$ 的大量 酸性矿坑废水. 同时, 媒矿石堆淋溶液沿冲沟进入湖泊水体. 由于 Fe、Mn 在湖底的积累, 近年 来每年有 2 个月支右的时间出现水体 Fe、Mn 浓度增高的水质恶化现象.

为认识阿哈湖水质变化机理.项目组于 1992 年 11 月至 1993 年 9 月在湖区不同点淕月采 集分层水样,并于冬季(1992 年11月)和秋季(1993 年 9 月)利用沉段物-水界面采样装置采集 沉积物柱芯和上覆界面水柱的样品 ${ }^{[5]}$. 沉积物孔隙水样品系在氮气中用离心法提取. 室内分析 分别采用了水化学全分析、离子选择电极、离子色谱、元素分析、原子吸收光谱、比色和容量法.

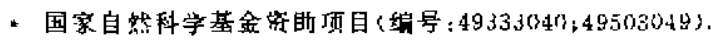

收稿日期; $1996-04-08$; 收到修改揖日期; $1996-07-12$.

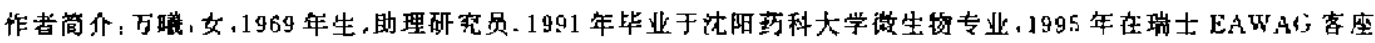
研究. 主要从事环境消生场地理字研究。 


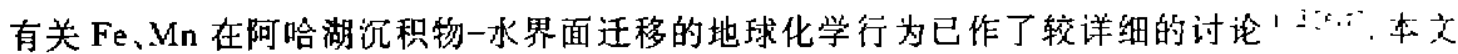
拟从生物地球化学角度对 $\mathrm{Fe} 、 \mathrm{Mn}$ 的沉积后再迁移效应进行分析.并探讨控制 $\mathrm{Fe} 、 \mathrm{Mn}$ 二次释 放的生物地球化学途经,为改善水质提供理论依据.

$1 \mathrm{Fe}^{2+}$ 在沉积物扩散边界层中被抑制及溶解态 $\mathrm{Mn}^{2+}$ 的迁移

\section{1 湖水 $\mathrm{Fe} 、 \mathrm{Mn}$ 浓度的季节性变化}

图1系1992 年11月至 1993 年 9 月对湖水分层采样的分析结果. 该图反映了 1992 年水 质变化周期中 $\mathrm{Fe} 、 \mathrm{Mn}$ 浓度回落阶段及 1993 年 7 月起的上升趋势. 由图 1 可以看出: 深水湖区 水体 $\mathrm{Mn}$ 度在大部分时间内处于较低水平 $(<0.01 \mathrm{mg} / \mathrm{L}), 7-9$ 月.下层水体的 $\mathrm{Mn}$ 浓度呈 增高趋势. 水体 $\mathrm{Fe}$ 浓度与 $\mathrm{Mn}$ 浓度的变化趋势相似, 但下层水体 $\mathrm{Fe}$ 浓度增高的起始时间晚于 $\mathrm{Mn}$; 回落的时间早于 $\mathrm{Mn}$; 增高的幅度也低于 $\mathrm{Mn}$. 此现象反映出 $\mathrm{Mn}$ 在沉积物一水界面的地球 化学循环较 $\mathrm{Fe}$ 剧烈.
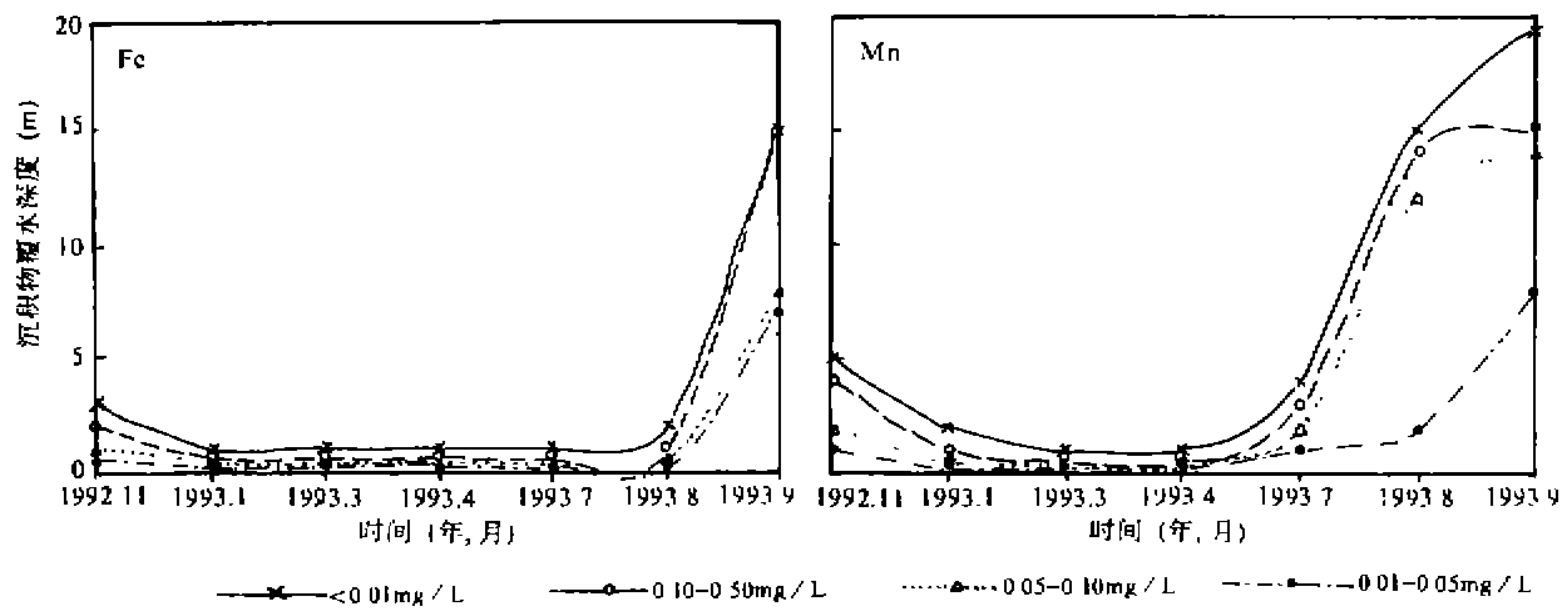

时间(年，月)

图 1 阿哈湖坝前水体 $\mathrm{Fe} 、 \mathrm{Mn}$ 浓度季节变化

Fig. 1 Seasonal variation of $\mathrm{Fe}, \mathrm{Mn}$ in Lake Aha

\section{2 水体物理化学条件的季节性变化}

湖水 $\mathrm{Fe} 、 \mathrm{Mn}$ 浓度的季节性变化与水体物理化学背景条件的变化有关. 图 2 显示 7-9月 湖体水温升高, 水体 $\mathrm{pH} 、 \mathrm{DO}$ 和 $\mathrm{SO}_{4}{ }^{2-}$ 浓度降低的情况. 7-9 月湖体水温明显升高、上、下层水 温之差近 $10 \mathrm{C}$, 并形成明显的温跃现象 (图 2a). 3.4 月湖水 $\mathrm{pH}$ 值较 1 月份明显升高.7 月份起 中下层水体出现碱度降低的趋势; 特别是 8,9 月, 中层水体的酸化 $(\mathrm{pH}<7)$ 势头更加明显(图 $2 b)$, 与水温和酸碱度变化相似, $7-9$ 月中下层水体溶解氧饱和百分比降至很低水平 $\left(20^{\circ}, \mathrm{i}\right)$ （图 2c).7--9 月水体 $\mathrm{SO}_{4}{ }^{2}$ 降低, 特别是 7.8 月份上层水体 $\mathrm{SO}_{4}{ }^{2-}$ 浓度降低更明显 (图 $2 \mathrm{~d}$ ). 水 体 $\mathrm{SO}_{4}{ }^{2-}$ 浓度降低既可能与雨季降水稀释作用有关, 也可能是硫酸盐还原作用的结果.

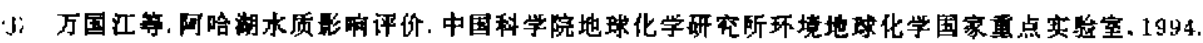

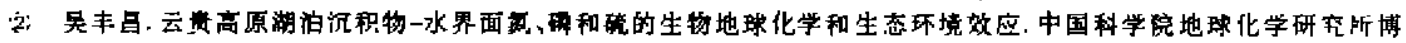
古论文, 1995 . 

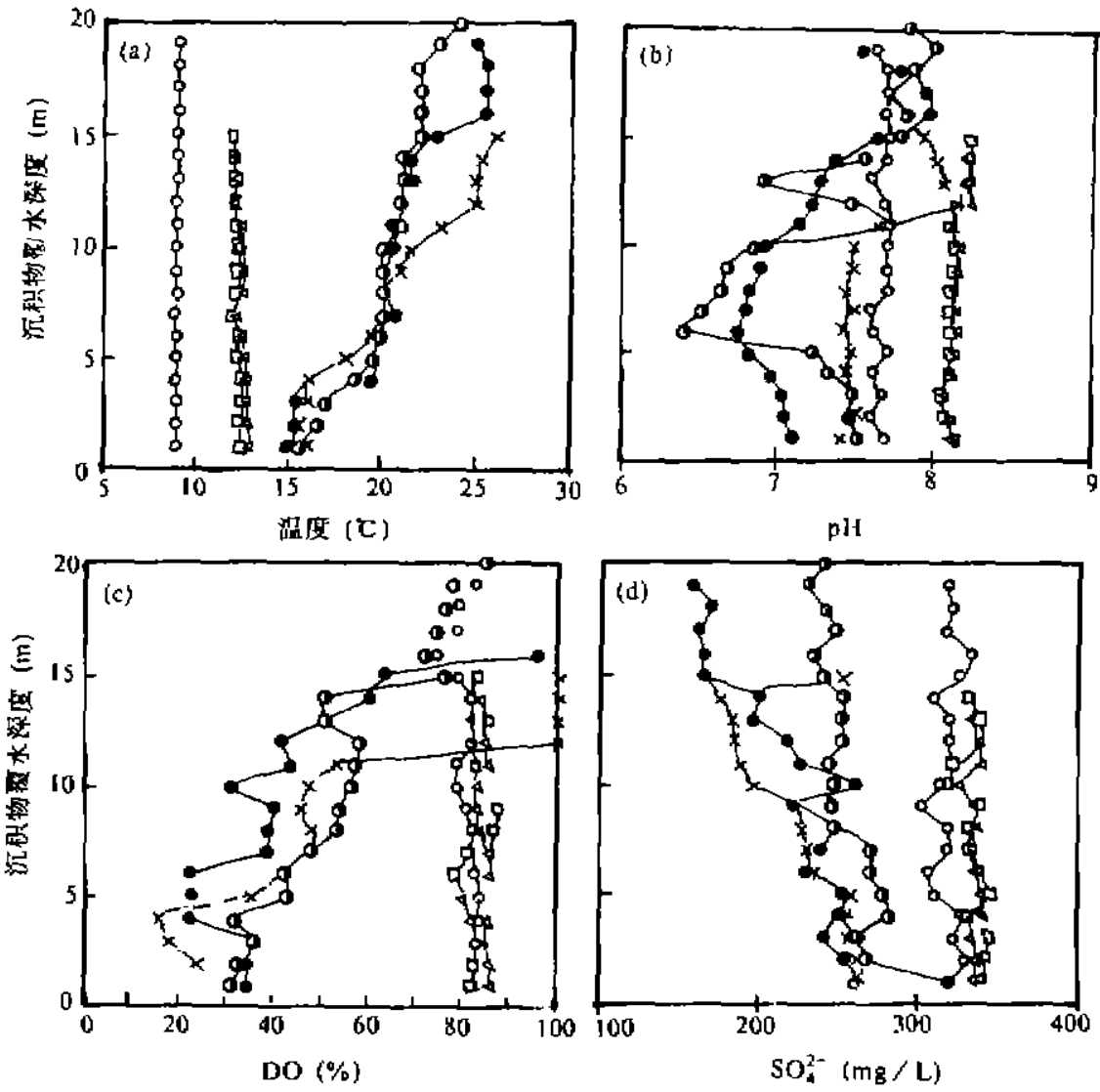

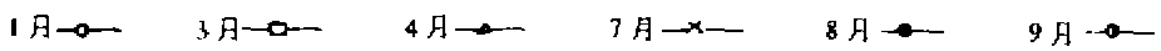

图 2 阿哈湖水体温度、些、DO 及 $\mathrm{SO}_{4}{ }^{2}-$ 浓度垂直变化

Fig. 2 Vertical varitions of temperature, $\mathrm{pH}, \mathrm{DO}$ and $\mathrm{SO}_{4}{ }^{2-}$ in Lake Aha

\section{1. $3 \mathrm{Fe}^{2+} 、 \mathrm{Mn}^{2+}$ 在沉积物一水界面迁移行为的差异}

在沉积物悬浮层及其以上界面水中、 $\mathrm{Fe}$ 的固一液分配系数 $\left(K_{d}\right)$ 在秋季明显低于冬季,说明 秋季湖底缺氧时 $\mathrm{Fe}^{2+}$ 可能自沉积物向上覆水体扩散. 但其 $K_{d}$ 的增大趋势显示出 $\mathrm{Fe}^{\mathrm{i}-}$ 的界面 皅环受扩散边界层抑制 ${ }^{\left[{ }^{[}\right]}$. 在相同层位 $\mathrm{Mn}$ 的 $K_{d}$ 值不仅秋季较冬季大幅度降低,而且还低于 悬浮层以下沉积物的 $K_{d}$. 说明 $\mathrm{Mn}^{2+}$ 自沉积物向上覆水体强烈释放 (图 3). 此外,关于沉积物水界面 $\mathrm{Fe} 、 \mathrm{Mn}$ 的沉降通量和扩散通量关系的概算 ${ }^{[3]}$, 也证实了阿哈湖沉积物一水界面上 $\mathrm{Fe}^{2+}$ 话环受抑制及 $\mathrm{Mn}^{2+}$ 䀠环的激烈进行.

$\mathrm{Fe}^{3+} \rightarrow \mathrm{Fe}^{2+} 、 \mathrm{Mn}^{4+} \rightarrow \mathrm{Mn}^{2+}$ 和 $\mathrm{SO}_{4}{ }^{2-} \rightarrow \mathrm{S}^{2-}$ 均产生于特定的氧化还原条件下. 氧化还原平衡 模式表明.水溶液中 $\mathrm{Fe}^{2+} 、 \mathrm{Mn}^{2+}$ 浓度随电子活度值 $\left(P_{s}\right)$ 的降低而升高. 在 $\mathrm{CaSO}$ 、饱和溶液中, 当 $\mathrm{pH}<7$ 时. $\mathrm{SO}_{4}{ }^{2-}$ 被还原为 $\mathrm{S}^{2-}$, 当 $\mathrm{pH}=7$ 时, 需要 $P_{\mathrm{s}}<-3, \mathrm{SO}_{4}{ }^{2-}$ 才明显地还原为 $\mathrm{S}^{2-[\mathrm{a}}$ ．

2 沉积物中硫酸盐的还原作用及 $\mathrm{Mn}$ 的还原过程

\section{1 沉积物硫酸盐的还原作用深度的变化}

沉积物生物氧化作用的热力学氧化还原方程包括 ${ }^{[3]}$; 

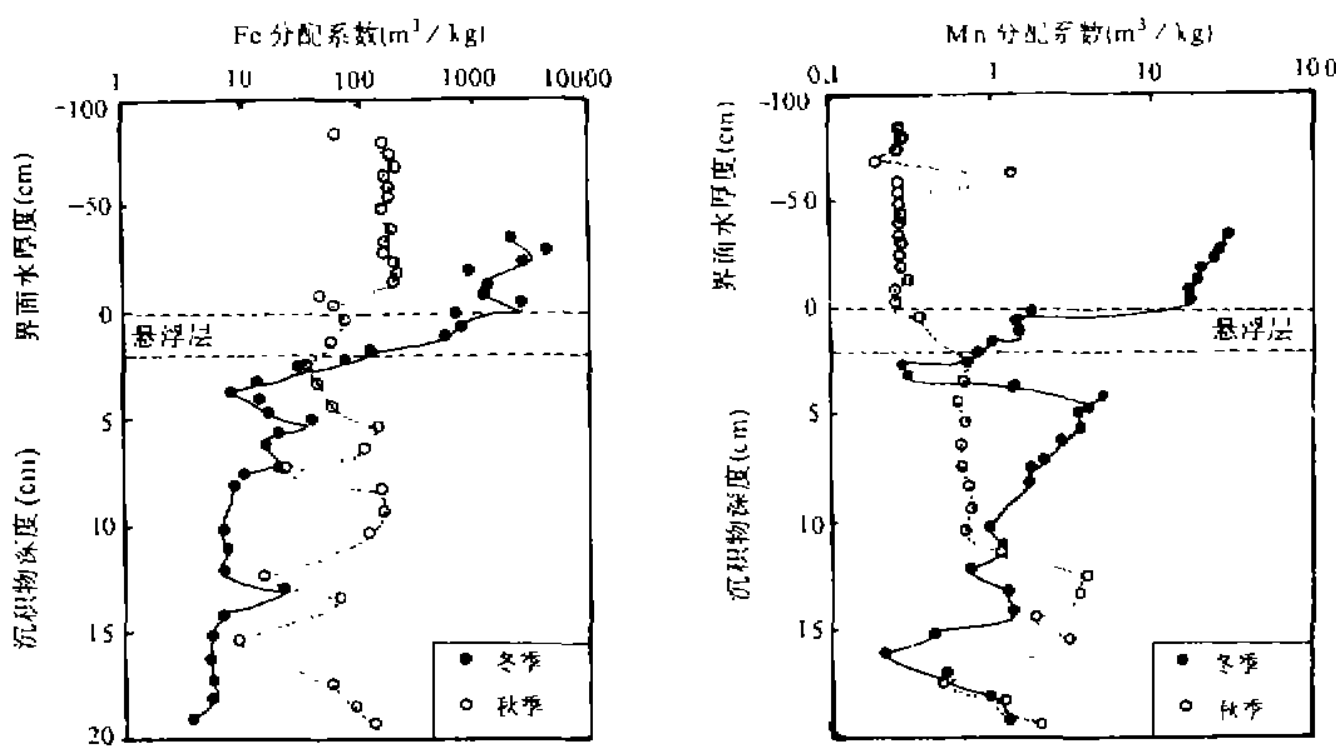

图 3 阿哈湖沉积物一水界面附近 Fe、Mn 的固-㳸分配系数的垂直变化

Fig. 3 Vertical variation of partıcle-water distributın coefficient of $\mathrm{Fe}, \mathrm{Mn}$ un the rediment-water interface of Lake Aha

(I) $\mathrm{H}_{2} \mathrm{~S}$ 的氧化作用:

$$
\mathrm{H}_{2} \mathrm{~S}+2 \mathrm{O}_{2} \rightarrow \mathrm{SO}_{4}{ }^{2}-2 \mathrm{H}^{+}
$$

(2) $\mathrm{NO}_{3}{ }^{-}$的还原作用: $\mathrm{BM}+84.8 \mathrm{NO}_{3}{ }^{-} \rightarrow 106 \mathrm{HCO}_{3}{ }^{-}+16 \mathrm{NH}_{4}{ }^{+}+\mathrm{HPO}_{4}{ }^{2-}+42.4 \mathrm{~N}_{2}-7,2 \mathrm{H}^{+}$ (3) $\mathrm{MnO}_{2}$ （固)的还原作用:

$$
\mathrm{BM}+212 \mathrm{MnO}_{21} ;{ }^{+}+332 \mathrm{H}^{+} \rightarrow 106 \mathrm{HCO}_{3}{ }^{-}+16 \mathrm{NH}_{4}{ }^{+}+\mathrm{HPO}_{4}{ }^{2-}+212 \mathrm{Mn}^{2+}
$$

(4) $\mathrm{FeO}(\mathrm{OH})$ ，困，的还原作用:

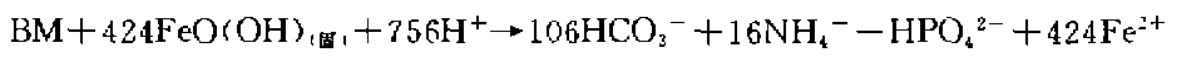

(5) $\mathrm{SO}_{4}{ }^{2-}$ 的还原作用: $\mathrm{BM}+53 \mathrm{SO}_{4}{ }^{2-}+14 \mathrm{H}^{+} \rightarrow 106 \mathrm{HCO}_{3}{ }^{-}+16 \mathrm{NH}_{4}{ }^{+}+\mathrm{HPO}_{4}{ }^{2-}+53 \mathrm{H}_{2} \mathrm{~S}$

以上各氧化还原方程式中, $\mathrm{H}_{2} \mathrm{O}$ 被忽略: $\mathrm{BM}$ 代表按雷德菲尔(Redfield)化学计算的生物 量: $\left(\mathrm{CH}_{2} \mathrm{O}\right) 106\left(\mathrm{NH}_{3}\right) 16 \mathrm{H}_{3} \mathrm{PO}_{4}$.

阿哈湖深水湖区沉积物柱芯具不规则的灰黑交替纹理结构,表层沉积物性质随季节而变 化. 冬春, 沉积物顶部悬浮县微粒为黄褐色, 厚度约 $2 \mathrm{~cm} ; \mathrm{Fe} 、 \mathrm{Mn}$ 处于氧化状态. 含量分别为 120 和 $2 \%$ 氧化层以下沉积物为灰黑色. 即 $\mathrm{Fe}$ 的硫化层. 当秋季下层水体缺氧时, 沉积物顶 部微粒悬浮层颜色变黑, 处于还原状态; 硫化层的上边界移至沉积物顶部; $\mathrm{Fe} 、 \mathrm{Mn}$ 含量分别降

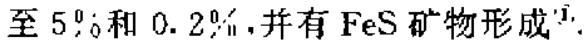

\section{2 磁酸盐还原作用的过程}

$\mathrm{SO}_{4}{ }^{2-}$ 是阿哈湖水的主要阴离子, 冬春季节含量为 $2.5-3 \mathrm{mmol} / \mathrm{L}$ ，约为湖水饱和溶㳸氧 摩尔旅度的 10 倍, $\mathrm{SO}_{4}{ }^{2-}$ 渗透到沉积物中的深度世比氧深 10 多倍. 坝前深水湖区沉积物顶部

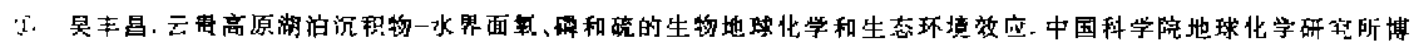
士论交.1995. 
孔腙水中 $\mathrm{SO}_{4}{ }^{2-}$ 浓度为 $1.6 \mathrm{mmol} / \mathrm{L}$; 在沉积物 $6 \mathrm{~cm}$ 深度以下降至 $0.1 \mathrm{mmol} / \mathrm{L}$ 并迅速耗尽. 从 而限制了硫酸盐还原作用进行的深度(图 4). 缺氧季 节、湖水 $\mathrm{SO}_{4}{ }^{2}-$ 含量略有下降, 溶解氧饱和百分含量 由上层湖水的 $80^{\circ}$ 。降至下层滞水带的 $20^{\circ}$ 左右、缺 氧季节还原菌产生的硫酸盐还原作用可能上移到沉 积物一水界面中进行.

由图 4 还看到, 冬春季节, 沉积物一水界面以下孔 隙水中的 $\mathrm{NO}_{3}{ }^{-}$的浓度急速下降. $\mathrm{NO}_{3}^{-}$的还原作用 仅在沉积物顶部界面处进行. $\mathrm{MnO}_{2 !}$ ， $\mathrm{FeO}(\mathrm{OH})$ ， 固 等的还原作用发生在悬浮是以下、6 $\mathrm{cm}$ 深度以上.当 缺氧季节、 $\mathrm{Fe} 、 \mathrm{Mn}$ 还原作用必然上移至沉积物一水 界面、导致是浮层微粒中 Fe、Mn 的含量降低.

脱硫弧菌属细菌是沉积物硫酸盐还原过程中起 主要作用的微生物. 这类硫酸盐还原细菌存在的基 本条件是: 还须环境、有 $\mathrm{SO}_{4}{ }^{2-}$ 存在及适当的能源供 给. 这 3 个条件在很大程度上決定了环境中硫酸盐 还原细菌的生长和代谢作用，当沉积物孔隙水中硫 酸盐浓度随深度增加而降低, 并有硫化物富集时, 便 证实了沉积物中存在硫酸盐还原细菌的活动 [s].

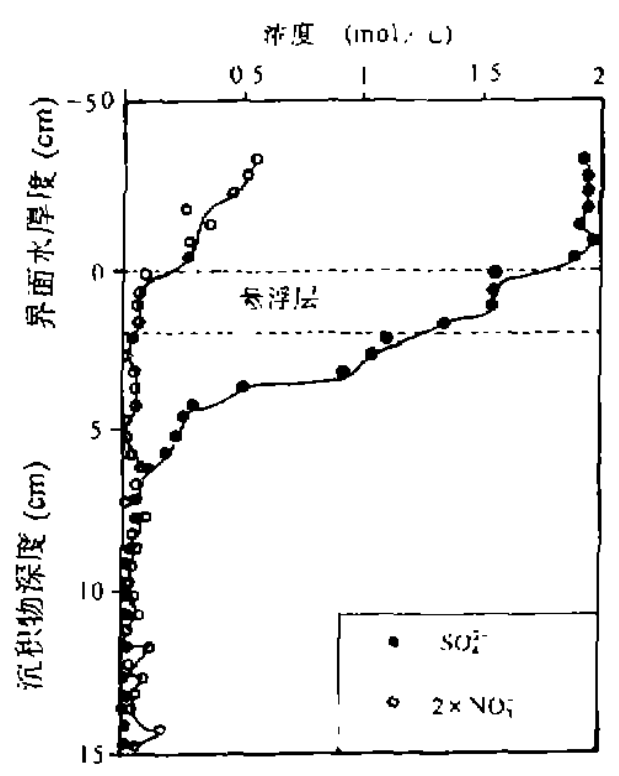

图 4 阿怡湖沉积物一水界面附近 $\mathrm{SO}_{4}{ }^{2-}$ 及 $\mathrm{NO}_{3}{ }^{-}$㳖度的垂直剖面

Fig. 4 Vertical profile of $\mathrm{SO}_{4}{ }^{-}$and $\mathrm{NO}_{3}^{-}$in the sediment-water interface of Lake Aha

脱硫张菌是严格厌氧的能动杆菌、以利用湖内 沉积物中有机物. 如乳酸盐、C-4二羧酸盐、丙酮酸盐等为碳源和能源. 此类细菌有不完全三 俊酸循环的酶系统, 有机碳被氧化不完全而作为 $\mathrm{CH}_{3} \mathrm{COO}^{-}$排出. 同时 $\mathrm{SO}_{4}{ }^{2-}$ 等作为最终电子 受体在厌氧条件下还原:

$$
\begin{gathered}
2 \mathrm{CH}_{3} \mathrm{CHOHCOO}+\mathrm{SO}_{4}{ }^{2-} \rightarrow 2 \mathrm{CH}_{3} \mathrm{COO}^{-}+2 \mathrm{HCO}_{3}{ }^{-}-\mathrm{H}_{2} \mathrm{~S} \\
4 \mathrm{H}_{2}-\mathrm{SO}_{4}{ }^{--}+2 \mathrm{H}^{+} \rightarrow 4 \mathrm{H}_{2} \mathrm{O}-\mathrm{H}_{2} \mathrm{~S}
\end{gathered}
$$

吸收 $\mathrm{Fe}$ 是微生物的一个基本功能. $\mathrm{Fe}$ 细菌在阿哈湖沉积物一水界面中对 $\mathrm{Fe}$ 的凝聚、沉淀 起重要作用. 当沉积物中被还原的 $\mathrm{Fe}^{i+}$ 进入孔隙水后, 很可能因硫弧菌作用形成 $\mathrm{FeS}$, 万至 $\mathrm{FeS}_{2}$ 沉淀; 也可能在中性 $\mathrm{pH}$ 的 $\mathrm{Fe}$ 细菌作用下.被氧化成 $\mathrm{Fe}^{3+}$ 而沉淀或进入有机鳌合物中.由 此可分析说明.阿哈湖缺氧季节, 硫酸盐的还原作用制约了溶解态 $\mathrm{Fe}^{2+}$ 向上覆水体扩散.

\section{$2.3 \mathrm{Mn}$ 氧化-还原作用的过程}

$\mathrm{Mn}^{2+}$ 的氧化作用需要分子氧与: $2 \mathrm{Mn}^{2+}+\mathrm{O}_{2}+4 \mathrm{OH}^{-} \rightarrow 2 \mathrm{H}_{2} \mathrm{O}+2 \mathrm{MnO}_{2}$, 当 $\mathrm{Mn}^{2+} 、 \mathrm{O}_{2}$ 或 $\mathrm{OH}^{-}$浓度增高时, 上述反应向右进行; 但当微喜氧菌属存在时, 低 $\mathrm{O}_{2}$ 也可使 $\mathrm{Mn}^{2}$-氧化. 特别 是生物学的氧化作用发生在 $p H 6.5-7.5$ 的条件下.由于微生物对有机碳氧化作用的影响.湖 泊沉积物中的有机碳越多. 则严重缺氧昌致 $\mathrm{Mn}^{4+}$ 的还原越激烈. 这一机理促成了阿哈湖季节 性缺氧时 $\mathrm{Mn}$ 自沉积物向水体激烈释放.

虽然在 $\mathrm{pH}$ 降低及还原状态下沉积物中 $\mathrm{Mn}^{4+}$ 趋向于形成可溶性 $\mathrm{Mn}^{2+}$, 并通过孔腺水向 上覆水体扩散.但许多生物都能在 $\mathrm{Mn}$ 含量低时聚集 $\mathrm{Mn}^{\left[{ }^{[9]}\right.}$. 天然淡水湖底沉积物孔烸水中可 以识别出 $10^{\circ}-10^{\circ} \mathrm{mL}^{-1}$ 的凝聚 $\mathrm{Mn}$ 细菌。尽管 $\mathrm{Mn}$ 细菌与天然沉积物伴生可能是沉积作用的 
结果,而非沉淀的原因; 但是沉积物中大量活性细菌的存在意味着存在一种生物地球化学作用 促使 $\mathrm{Mn}$ 细菌凝聚。作者试图通过 $\mathrm{Mn}$ 细菌凝集作用的研究来控制 $\mathrm{Mn}$ 的季节性扩散效应.

\title{
参考文 献
}

1 Davison W, Iron and Manganese in lakes. Earth-Sctemue Reveats,1996,34:119-163

2 Stumm $W$ and B Sulzberger. The cycling of iron in natural environments, consideration based on laboratory studies of heterogeneous redox processes. Gevechimsa et Cormachumica Acta.1992,56, 3233-3257

3 Furrer $\mathrm{G}$ and B Wehrli. Brogeochemical processes at the sediment-water interface ; measurements and modeling. Appliced Liev chemistr $y, 1993,\{2): 117-119$

4 Jobnson C A, M Ulricb.L Sigg, et al. A matbernatical model of the Maganese cycle in a seasonally anoxic lakes. Limnul O Leurrogr.1991.36( 7$)_{1} 1415-1426$

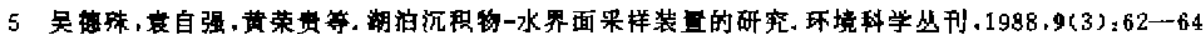

6 Wan G J, R G Huang, Z L Chen, et al. Screening effect of diffusive boundary layer in sediments of Lake Aba. Chinese .I of Lieachentistry, 1997,16 (In press)

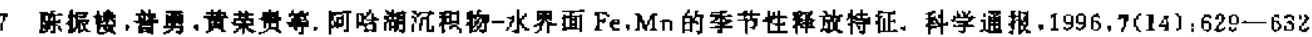

8 Stumm W and J J Morgan. Aquatic Chemistry. Yew York: John Wiley and Sons. 1981

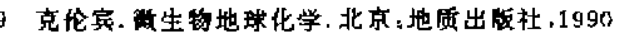

\section{BIOGEOCHEMICAL EFFECT ON POSTDEPOSITIONAL REMO- BILIZATION OF Fe - Mn IN LAKE AHA, GUIZHOU PROVINCE}

\author{
Wan X:1 Wan Guojiang ${ }^{2}$ Huang Ronggui ${ }^{2}$ Pu Yong ${ }^{2}$ \\ (1: Inttatute of Soil and Fertilizer, Chusese Academy of Agricultural Sciences, Bergang 100ng1, \\ 2: State Key Lab. of Enjeranmentul Geachemestry. Instutute of Geachentastry. CAS.Guiyung 550002]
}

\begin{abstract}
Lake Aha, located in the suburb of Guiyang, Guizhou Province, is a medium artificial reservoir with seasonally anoxic hypolimina. After a long-term accumulation.iron and manganese are enriched in the upper sediments. When waters of oplimnion and hypolimnion are anoxic in late summer and early autumn, $\mathrm{Fe}^{2+}$ and $\mathrm{Mn}^{2+}$ formed by biological respiration, are diffused upto the overlying waters from the sediments. However, the concentration of $\mathrm{Fe}^{2+}$ increases later and secreases earlier than that of $\mathrm{Mn}^{2+}$.

Generally, sulphate reduction takes place within $6 \mathrm{~cm}$ depth of the sediments. Whereas in the anoxic season, the reduction reaches the upper sediment, inhibiting the diffusion of $\mathrm{Fe}^{i+}$. Meanwhile, manganese oxidation needing molecular oxygen as catalyst, causes the violent diffusion of $\mathrm{Mn}^{2+}$. Because manganese can be accumulated by microorganism in natural fresh water. it is important to seek method to control manganese remobilization through manganese accumulated action .
\end{abstract}

Key Words Postdepositional remobilizaton of $\mathrm{Fe}-\mathrm{Mn}$, biogeochemical effect, Lake Aha 